\title{
Metabolic profiling of human follicular fluid identifies potential biomarkers of oocyte developmental competence
}

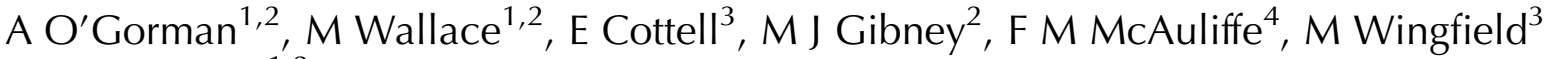 \\ and L Brennan ${ }^{1,2}$ \\ ${ }^{1}$ UCD School of Agriculture and Food Science, UCD Conway Institute, Belfield, Dublin, Ireland, ${ }^{2}$ UCD Institute of \\ Food and Health, Belfield, Dublin, Ireland, ${ }^{3}$ Merrion Fertility Clinic, National Maternity Hospital, Dublin, Ireland and \\ ${ }^{4}$ UCD Obstetrics and Gynaecology, School of Medicine and Medical Science, National Maternity Hospital, \\ Dublin, Ireland
}

Correspondence should be addressed to L Brennan at UCD School of Agriculture and Food Science, UCD Conway Institute, Belfield, Dublin, Ireland; Email: lorraine.brennan@ucd.ie

\begin{abstract}
The use of metabolomic based techniques to aid oocyte and embryo selection has gained attention in recent years. Previous work from our laboratory has demonstrated that the ${ }^{1} \mathrm{H}$ NMR-based metabolic profile of follicular fluid correlates with oocyte developmental potential. Patients undergoing IVF at the Merrion Fertility Clinic had follicular fluid collected at the time of oocyte retrieval. The fatty acid composition of follicular fluid from follicles where oocytes fertilised and developed into multi-cell embryos $(n=15)$ and from oocytes that fertilised normally but failed to cleave $(n=9)$ (cleaved vs non-cleaved) was compared. Statistical analysis was performed on the data using univariate and multivariate techniques. Analysis of the fatty acid composition revealed that there were nine fatty acids significantly different between follicular fluid from the cleaved and the non-cleaved sample groups. Of particular interest were the higher concentration of total saturated $(P=0.03)$ and the lower concentration of total polyunsaturated fatty acids in the non-cleaved sample group $(P=0.001)$. Random forest classification models were used to predict successful cleavage in follicular fluid samples producing models with errors rates of $<\mathbf{1 0} \%$. Receiver operating characteristic analysis demonstrated that the model had good predictability with an area under the curve of 0.96 . The panel of fatty acid biomarkers identified in this study indicates that the fatty acid composition of follicular fluid may be more predictive in comparison to other previously identified biomarkers. Following validation in a larger cohort, these biomarkers may have the potential to be used in fertility clinics to aid the selection of oocytes in the future.

Reproduction (2013) 146 389-395
\end{abstract}

\section{Introduction}

Despite the recent advances in IVF technology, only $32 \%$ of completed IVF cycles result in a clinical pregnancy (de Mouzon et al. 2010). In an attempt to optimise pregnancy rates, multiple embryos may be replaced per treatment cycle, increasing the number of multiple pregnancies, leading to a greater incidence of maternal and neonatal complications. The ability to choose the 'best' embryo for transfer would ultimately allow implantation of a single embryo of superior quality, thereby improving pregnancy rates and reducing the chances of a multiple pregnancy (Haggarty et al. 2006). At present, developmental rates and morphological assessment are the main methods used to determine embryo viability during IVF cycles, but these are relatively poor indicators of successful implantation (Sinclair et al. 2007, Singh \& Sinclair 2007).
Oocyte quality or developmental competence is intrinsic to the development of a viable embryo (Mtango et al. 2008). Common ovarian stimulation procedures used in assisted reproductive technology cycles may suppress the natural selection procedure and allow seemingly successful maturation of oocytes. However, these may have inherent compromised quality and may be destined to fertilisation failure, compromised embryo development or long-term consequences in vivo (Swain $\&$ Pool 2008). Therefore, it has been proposed that only a fraction of the oocytes retrieved in an IVF cycle have the potential to develop into a viable embryo destined for a live birth (Kovalevsky \& Patrizio 2005). Despite the importance of oocyte quality, current assessment of oocytes in the routine IVF laboratory is limited, and following fertilisation, the developmental potential of the embryo is estimated predominantly on the cell stage and morphology of the embryo, rather than the quality of 
the oocyte it was derived from Rienzi et al. (2011). Development of accurate parameters from non-invasive markers that predict oocyte developmental competence would increase the accuracy of embryo selection and could lead to oocyte selection methods that would aid in overcoming the ethical, legal and storage implications of current supernumerary frozen embryos (Kovalevsky \& Patrizio 2005).

Investigation of follicular fluid composition as a possible predictor of both oocyte developmental competence and embryo viability has increased in recent years (Wu et al. 2007, Berker et al. 2009, Revelli et al. 2009, Piñero-Sagredo et al. 2010, Wallace et al. 2012). Follicular fluid contains important metabolites that can be critical for oocyte growth and development and provides important information about the growth and differentiation of the follicle (Edwards 1974).

Metabolomics is the systematic study of metabolites as small-molecule biomarkers that represent the functional phenotype in a cell, tissue or organism. It has proven to be a useful technology for analysis of metabolism of several biological systems and has been used in the study of human embryos (Botros et al. 2008, Nagy et al. 2008, Scott et al. 2008, Vergouw et al. 2008, 2012) and oocytes (Singh \& Sinclair 2007, Nagy et al. 2009) via analysis of culture media. Nuclear magnetic resonance (NMR) spectroscopy has been previously used in order to profile metabolites extensively in follicular fluid (Piñero-Sagredo et al. 2010). Work from our laboratory has demonstrated that the ${ }^{1} \mathrm{H}$ NMR-based metabolic profile of follicular fluid correlates with oocyte developmental potential, with a number of metabolites (e.g. glucose, lactate, choline, phosphocholine, proline, leucine/isoleucine, glutamine and HDL) identified as being important indicators of whether a fertilised oocyte will divide and develop into an early cleavage stage embryo or fail to cleave (Wallace et al. 2012).

Although a number of studies have looked at the free fatty acid and triglyceride composition of follicular fluid (Robker et al. 2009, Jungheim et al. 2011b, Yang et al. 2012), to the best of our knowledge, a gas chromatography/mass spectroscopy (GC/MS)-based approach to identify fatty acid markers of oocyte quality in human follicular fluid has not been carried out. A major advantage of this technique is the associated high sensitivity; therefore, it may detect metabolites that are present in a concentration below the detection limit of other techniques such as ${ }^{1} \mathrm{H}$ NMR spectroscopy (Bedair \& Sumner 2008, Lei et al. 2011). Therefore, the objective of this study was to use a GC/MS metabolomic approach to profile the composition of follicular fluid from follicles where oocytes fertilised and developed into early cleavage stage embryos $(n=15)$ and from oocytes that fertilised normally but failed to cleave $(n=9)$. A secondary aim was to apply multivariate statistical analysis to the data in an attempt to identify potential markers of oocyte developmental competence.

\section{Materials and methods}

\section{Sample collection}

Ethical approval for this study was obtained from the Human Research Ethics Committee, University College Dublin and the Research Ethics Committee of the National Maternity Hospital (Dublin). As part of a larger study, 58 patients undergoing IVF treatment at the Merrion Fertility Clinic (Dublin), were recruited and provided written informed consent (Wallace et al. 2012). Patients either received a standard GNRH agonist (Buserelin) regime starting on day 21 of a spontaneous menstrual cycle or a standard antagonist regime (Ganirelix). Recombinant FSH stimulation was initiated once downregulation was confirmed via ultrasound and serum oestradiol $\left(E_{2}\right)$ measurements or on day 2 or 3 for antagonist cycles. Further stimulation doses were determined according to standard criterion for follicular maturation, assessed by ultrasound and serum $E_{2}$ measurements. Choriogonadotropin alfa $(6500 \mathrm{IU})$ was administered when at least three follicles had reached a diameter of $\geq 18 \mathrm{~mm}$.

Oocytes were collected by transvaginal ultrasound-guided needle aspiration of the follicles under deep conscious sedation. At the time of oocyte retrieval, the follicular fluid from one to three follicles between the sizes of 14 and $24 \mathrm{~mm}$ were collected per patient. To avoid the collection of bloodcontaminated follicular fluid samples, a midstream aspirate was collected for each patient and checked for blood contamination both visually and via spectrophotometric analysis (Levay et al. 1997). In cases where two samples were collected, the needle was rinsed between aspirates. Samples were then centrifuged at $10000 \mathrm{~g}$ for $10 \mathrm{~min}$ and the supernatant was aliquoted and stored at $-80{ }^{\circ} \mathrm{C}$ until further analysis. Oocytes collected from the follicles where fluid was retained were individually cultured in order to track their outcome. On the day after oocyte retrieval and insemination (day 1 ), each oocyte was examined for evidence of fertilisation. Those that were found to have two pronuclei were transferred to $30 \mu \mathrm{l}$ micro-drops of Quinns advantage cleavage medium (SAGE BioPharma, Inc., Bedminster, NJ, USA) and overlaid with paraffin oil for culture until embryo transfer on day 2 .

A total of 108 follicular fluid samples were collected and based on the developmental competence of the oocyte (Wallace et al. 2012), a subset of these samples was selected for analysis in this study: this subset included the 2-cell embryo group where there was sufficient follicular fluid for further analysis and the non-cleaving fertilised group. Hence, follicular fluid from follicles where the oocyte fertilised and developed into an early cleavage stage embryo $(n=15)$ as observed on day 2 and from follicles where the oocyte fertilised but failed to cleave $(n=9)$ was analysed. These two classes of samples are further referred to as cleaved and non-cleaved respectively. Follicular fluid samples from 19 patients were used in this study and the characteristics of the study population are given in Table 1.

\section{Metabolite extraction and data analysis}

Follicular fluid samples were thawed on ice prior to analysis. Organic compounds were analysed as follows: $300 \mu \mathrm{l}$ follicular 
Table 1 Baseline characteristics of the study population.

\begin{tabular}{lcc}
\hline Patient characteristics & $\begin{array}{c}\text { Cleaved group } \\
(n=15 \text { samples })\end{array}$ & $\begin{array}{c}\text { Non-cleaved group } \\
(n=9 \text { samples })\end{array}$ \\
\hline No. of patients & $12^{\mathrm{a}, \mathrm{b}}$ & $9^{\mathrm{b}, \mathrm{c}}$ \\
Age (years) & $36.7(3.8)$ & $37.7(2.1)$ \\
$\mathrm{BMI}\left(\mathrm{kg} / \mathrm{m}^{2}\right)$ & $24.2(3.4)$ & $22.6(2.6)$ \\
$\mathrm{FSH}(\mathrm{U} / \mathrm{l})$, cycle day 2 & $7.1(2.5)$ & $7.3(2.3)$ \\
$\mathrm{E}_{2}(\mathrm{pmol} / \mathrm{l})$ on day of Hcg & $4836(2079.4)$ & $4090.5(1506.8)$ \\
\hline
\end{tabular}

Values are averages \pm s.D.

${ }^{a}$ Twelve patients provided 15 follicular fluid samples. Nine patients provided one follicular fluid sample each and three patients provided two follicular fluid samples each $(n=15)$. ${ }^{\mathrm{b}}$ Two patients provided a sample each in both the cleaved and the non-cleaved groups. Overall total number of patients: $19 .{ }^{\mathrm{C}}$ Nine patients provided one follicular fluid sample each $(n=9)$.

fluid were combined with $50 \mu$ l heptadecanoic acid (C17:0; $2 \mathrm{mg} / \mathrm{ml}$ methanol) as an internal standard and extracted using a 1:2 mixture of chloroform:methanol based on the method of Bligh \& Dyer (1959). Extracts were derivatised by methylation using methanolic $\mathrm{BF}_{3}$. Derivatives were re-suspended in $200 \mu \mathrm{l}$ hexane and analysed by GC/MS.

The GC/MS system comprised an Agilent 7890A GC coupled with a 5975C ion-trap MS running in electron impact (EI) + mode. Chromatography was performed on an Agilent HP-5ms CP-sil 8 CB low bleed/MS capillary column (length $30 \mathrm{~m}$, diameter $0.25 \mathrm{~mm}$ and film thickness $0.25 \mu \mathrm{l}$ ) using helium at $1.2 \mathrm{ml} / \mathrm{min}$. Samples $(1 \mu \mathrm{l})$ were injected into a programmed temperature ramp in splitless mode. The GC temperature was initially $70^{\circ} \mathrm{C}$ for $2 \mathrm{~min}$, increased to $190^{\circ} \mathrm{C}$ at $15^{\circ} \mathrm{C} / \mathrm{min}$ for $1 \mathrm{~min}$, then increased to $230{ }^{\circ} \mathrm{C}$ at $5{ }^{\circ} \mathrm{C} / \mathrm{min}$ for $5 \mathrm{~min}$ and finally raised to $320^{\circ} \mathrm{C}$ at $20^{\circ} \mathrm{C} / \mathrm{min}$ for $5 \mathrm{~min}$. The GC/MS interface temperature was $220^{\circ} \mathrm{C}$. MS acquisition conditions were El ionisation of $70 \mathrm{eV}$, solvent delay of $1 \mathrm{~min}$, source temperature of $230{ }^{\circ} \mathrm{C}$ and mass range of 45-650 amu (atomic mass unit) at 2 scans/s.

Calibration was achieved by comparison of peak areas for fatty acids with reference to a known standard (Supelco 37 compound mix, Supelco, Poole, UK) using Agilent Chemstation (MSD E.02.00.493) and by comparison of their mass spectra with those in the NIST library 2.0. Automatic peak detection was carried out with Agilent Chemstation MSD. Mass spectra deconvolution was performed with the Automated Mass Spectral Deconvolution and Identification System (AMDIS, version 2.65). Peaks with a signal-to-noise ratio $(\mathrm{S} / \mathrm{N})$ lower than 30 were rejected, which is an acceptable level to avoid false positives as reported by Norli et al. (2010). To obtain accurate peak areas for internal standard and specific peaks/compounds, one quant mass for each peak was specified as the target ion and three masses were selected as qualifier ions. Each data file was then manually analysed for false positives/negatives in Agilent Chemstation. A matrix table with the concentration of each selected metabolite in each of the samples was produced complete with sample information and used for multivariate statistical analysis. Concentrations given for fatty acids are expressed as percentages \pm s.D.

\section{Statistical analyses}

Processed data were subjected to univariate and multivariate statistical analyses using the statistical package $R$ (version 2.14.1). The analysis of the GC/MS data involved the following steps:

i) A preliminary observation of the data using principal component analysis (PCA) to identify separation between the datasets and outliers as appropriate. Prior to PCA analysis, the data were scaled with a unit variance scaling.

ii) Modelling the data using random forests (RF) was performed to a) discriminate between cleaved and noncleaved samples and b) to identify important fatty acids that could be used as markers to predict oocyte developmental potential, i.e. cleaved/non-cleaved. The number of trees fitted was 750 , and the number of random metabolites tried at each node of the tree was set at 20 after optimisation. RF models are usually made up of hundreds of decision trees, with each tree constructed from a bootstrap of the original data set. Generally, two thirds of the samples will be included in a bootstrap sample and one third will be left out (called out-of-bag samples or OOB samples). For more detailed information, please see references proposed by Breiman (Breiman 2001).

iii) Univariate statistics (ANOVA) were used to assess the ability of the identified fatty acids (as identified by the RF model), which may be used as predictors of oocyte developmental potential. Significance was assumed when $P<0.05$.

iv) Partial least squares discriminant analysis (PLS-DA) was performed using the Caret (classification and regression) package in $\mathrm{R}$. The data set was randomly split into a training and test set, with $60 \%$ of the data used to train the model and the remainder $(40 \%)$ to evaluate the model performance, i.e. the test set, with a $y$ matrix set up to provide information on class. Performance of the classification models was evaluated on the basis of percentage correct classification rates.

v) Receiver operating characteristic (ROC) analysis was performed using the digeR package (Fan et al. 2009) in R. The classification performance (sensitivity and specificity) of the samples was assessed by the area under the curve (AUC).

\section{Results}

\section{Composition of follicular fluid}

A total of 21 fatty acids were identified and quantified in the follicular fluid (Table 2). The most abundant fatty acids found in follicular fluid from cleaved samples were palmitic acid, arachidonic acid, linoleic acid, stearic acid and oleic acid. In the follicular fluid from noncleaved samples, the same five fatty acids were the most abundant, although their order was different: palmitic acid, oleic acid, linoleic acid, arachidonic and stearic acid. From the 21 identified fatty acids, nine were statistically different between the two groups (Table 2). In addition, the total percentage of saturated fatty acids (SFA) in the follicular fluid was significantly higher in the non-cleaved samples, while the total percentage of polyunsaturated fatty acids (PUFAs) was lower in this group compared with the cleaved group (Table 2). 
Table 2 Fatty acid composition (percentage of total fatty acids) of the follicular fluid from embryos that cleaved $(n=15)$ and those that failed to cleave after fertilisation $(n=9)$.

\begin{tabular}{|c|c|c|c|}
\hline Fatty acid & Cleaved & Non-cleaved & $P$ value \\
\hline Total fatty acids $(\mu \mathrm{g} / \mathrm{ml})$ & $540.31 \pm 202.82$ & $646.76 \pm 50.60$ & NS \\
\hline Myristic acid (C14:0) & $0.95 \pm 0.37$ & $0.89 \pm 0.13$ & NS \\
\hline $\begin{array}{l}\text { Palmitoleic acid } \\
\text { (C16:1) }\end{array}$ & $2.32 \pm 0.91$ & $1.77 \pm 0.28$ & NS \\
\hline Palmitic acid (C16:0) & $27.75 \pm 3.74$ & $38.26 \pm 6.84$ & $6.73 \times 10^{-5}$ \\
\hline $\begin{array}{c}\gamma \text {-Linolenic acid } \\
(\text { C18:3n6) }\end{array}$ & $0.2 \pm 0.07$ & $0.17 \pm 0.06$ & NS \\
\hline $\begin{array}{l}\text { Linolenic acid } \\
(\mathrm{C} 18: 3 \mathrm{n} 3)\end{array}$ & $0.02 \pm 0.01$ & $0.02 \pm 0.01$ & NS \\
\hline $\begin{array}{l}\text { Linoleic acid } \\
\quad(C 18: 2 \mathrm{n} 6)\end{array}$ & $13.97 \pm 1.49$ & $12.4 \pm 3.71$ & NS \\
\hline Oleic acid (C18:1n9c) & $12.79 \pm 2.11$ & $13.6 \pm 1.97$ & NS \\
\hline Elaidic acid (C18:1n9t) & $0.66 \pm 0.24$ & $0.84 \pm 0.33$ & NS \\
\hline Stearic acid (C18:0) & $13.31 \pm 1.06$ & $10.58 \pm 2.98$ & $3.58 \times 10^{-3}$ \\
\hline $\begin{array}{l}\text { Arachidonic acid } \\
\text { (C20:4n6) }\end{array}$ & $15.69 \pm 3.29$ & $12.24 \pm 2.88$ & $1.63 \times 10^{-2}$ \\
\hline EPA (C20:5n3) & $0.87 \pm 0.47$ & $0.74 \pm 0.39$ & NS \\
\hline $\begin{array}{l}\text { cis-Eicosadienoic acid } \\
(\text { C20:2) }\end{array}$ & $0.36 \pm 0.12$ & $0.3 \pm 0.14$ & NS \\
\hline $\begin{array}{l}\text { cis-11-Eicosanoic acid } \\
\text { (C20:1) }\end{array}$ & $0.23 \pm 0.11$ & $0.26 \pm 0.08$ & NS \\
\hline DGLA (C20:3n6) & $2.75 \pm 0.61$ & $2.03 \pm 0.66$ & $1.24 \times 10^{-2}$ \\
\hline Arachidic acid (C20:0) & $0.19 \pm 0.03$ & $0.3 \pm 0.11$ & $9.29 \times 10^{-4}$ \\
\hline DHA (C22:6n3) & $6.37 \pm 1.8$ & $3.25 \pm 0.46$ & $4.5 \times 10^{-5}$ \\
\hline Erucic acid (C22:1n9) & $0.03 \pm 0.01$ & $0.05 \pm 0.03$ & 0.05 \\
\hline Behenic acid (C22:0) & $0.3 \pm 0.14$ & $0.39 \pm 0.25$ & NS \\
\hline $\begin{array}{l}\text { Tricosanoic acid } \\
\text { (C23:0) }\end{array}$ & $0.16 \pm 0.06$ & $0.24 \pm 0.09$ & $1.85 \times 10^{-2}$ \\
\hline Nervonic acid (C24:1) & $0.79 \pm 0.2$ & $1.05 \pm 0.59$ & NS \\
\hline $\begin{array}{l}\text { Lignoceric acid } \\
\text { (C24:0) }\end{array}$ & $0.29 \pm 0.09$ & $0.59 \pm 0.18$ & $1.58 \times 10^{-5}$ \\
\hline Total SFA & $42.95 \pm 5.5$ & $51.26 \pm 10.58$ & $3.4 \times 10^{-2}$ \\
\hline Total MUFA & $16.82 \pm 3.58$ & $17.58 \pm 3.28$ & NS \\
\hline Total PUFA & $40.23 \pm 7.85$ & $31.16 \pm 8.32$ & $1.0 \times 10^{-3}$ \\
\hline n-3 PUFA ${ }^{a}$ & $7.26 \pm 2.27$ & $4.01 \pm 0.87$ & $1.52 \times 10^{-2}$ \\
\hline n-6 PUFA ${ }^{b}$ & $32.61 \pm 5.46$ & $26.84 \pm 7.31$ & NS \\
\hline n-6:n-3 PUFA ratio & $4.79 \pm 1.44$ & $6.84 \pm 1.31$ & $2.1 \times 10^{-3}$ \\
\hline
\end{tabular}

Values are expressed as percentages (\%) \pm s.D. SFA, saturated fatty acids; MUFA, monounsaturated fatty acids; PUFAs, polyunsaturated fatty acids; EPA, eicosapentaenoic acid; ETE, eicosatrienoic acid; DHA, docosahexaenoic acid; DGLA, dihomo- $\gamma$-linolenic acid. $a_{n}-3$ PUFA $=\Sigma(C 18: 3 n 3+C 20: 5 n 3+C 22: 6 n 3)$.

${ }^{b} n-6$ PUFA $=\Sigma(C 18: 3 n 6+C 18: 2 n 6+C 20: 4 n 6+C 20: 3 n 6)$.

PCA revealed good separation between the two classes (cleaved and non-cleaved). The score plot PC1 vs PC2 is depicted in Fig. 1; these first two principal components accounted for 25 and 19\% respectively of the total variance.

\section{Prediction of oocyte developmental competence}

RF modelling was applied with the aim of discriminating between cleaved and non-cleaved samples and to identify specific fatty acids that could potentially be used as markers for predicting oocyte developmental competence. This resulted in a good classification model with an out-of-bag error rate of $8 \%$, i.e. $92 \%$ of samples were correctly classified. The top five most important variables for predicting oocyte developmental competence as indicated by the model were lignoceric acid, palmitic acid and arachidic acid, which were significantly increased in the non-cleaved group, and docosahexaenoic acid (DHA) and stearic acid, which were significantly increased in the cleaved group (Fig. 2). A PLS-DA model was also employed in an attempt to discriminate between the two sample groups. The model performed with good predictability, with $82 \%$ of samples correctly classified in the test set.

ROC analysis was performed and the AUC was used as an estimate of the predictive accuracy of the panel of biomarkers. Using the top five important metabolites as indicated by the RF model (Fig. 2), an AUC of 0.96 was obtained (Fig. 3). This favourable AUC value reveals that these fatty acids have a high predictive ability for oocyte developmental competence. This result corroborates with the previous models that were built (RF and PLS-DA), which all performed well in predicting oocyte developmental competence with low error rates.

\section{Discussion}

The most dominant fatty acids present in the human follicular fluid were palmitic acid, oleic acid, linoleic acid, arachidonic acid and stearic acid. Overall, the fatty acid composition of follicular fluid obtained from oocytes that developed into early cleavage stage embryos was significantly different from follicular fluid where the oocyte fertilised but failed to cleave (cleaved vs non-cleaved). Of the five most dominant metabolites, three were significantly different between the follicular fluid from the cleaved and the non-cleaved groups (palmitic acid, arachidonic acid and stearic acid). From the 21 fatty acids identified, seven were SFA, with five significantly different between the follicular fluid from

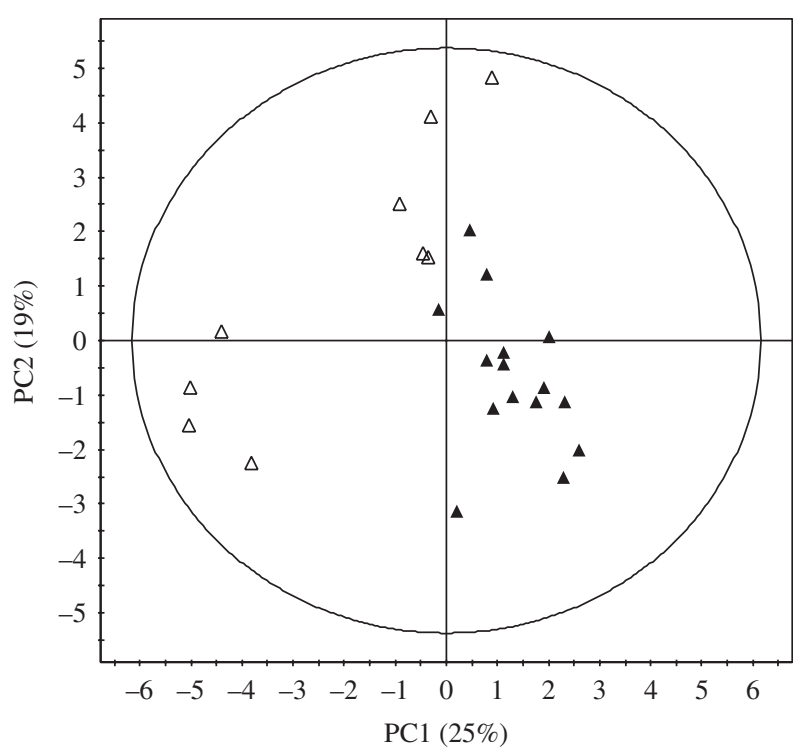

Figure 1 Principal component analysis (PCA) score plot of PC1 vs PC2 for follicular fluid samples from follicles where oocytes developed into early cleavage stage embryos (black triangles; $n=15$ ) and from follicular fluid where oocytes resulted in a fertilised egg that failed to cleave (open triangles; $n=9$ ). $R^{2}=0.6$, for a three-component model. 


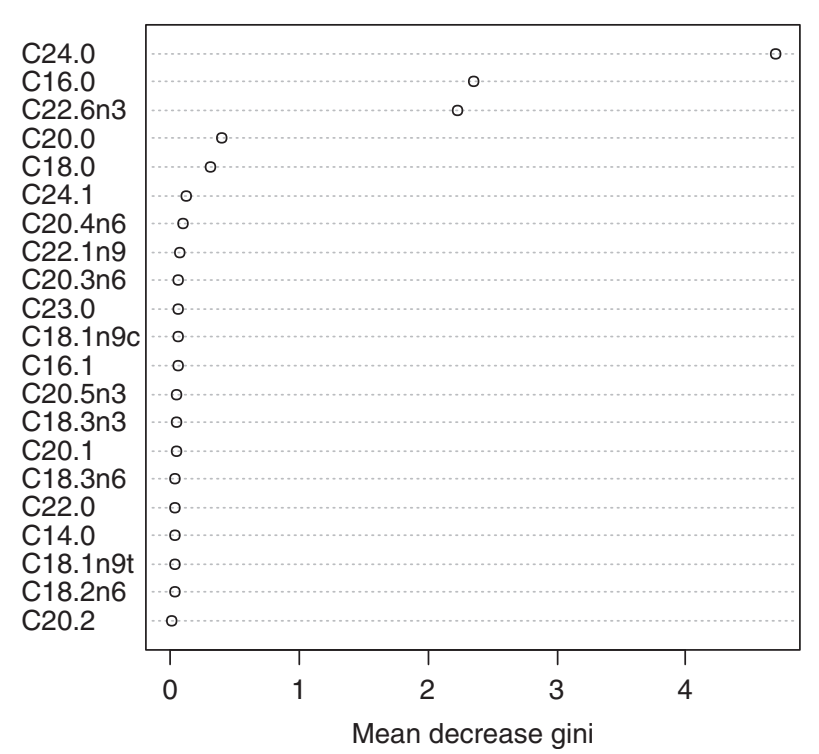

Figure 2 Variable importance plot (VIP) of the fatty acids that are important variables in the random forest (RF) model for predicting oocyte developmental competence.

the cleaved and the non-cleaved groups. Additionally, the total percentage of SFA in the follicular fluid was elevated in the non-cleaved group compared with follicular fluid from the cleaved group. Previous studies investigating the lipid composition of follicular fluid have found that increased levels of total free fatty acids and triglycerides are associated with negative effects on different reproductive outcomes (Robker et al. 2009, Aardema et al. 2011, Jungheim et al. 2011b, Yang et al. 2012). Recently, it has been demonstrated that exposure of mouse cumulus-oocyte complexes (COC) exposed to human follicular fluid with a high triglyceride and free fatty acid content during their maturation resulted in an increased oocyte lipid content, induction of endoplasmic reticulum stress markers and impaired oocyte nuclear maturation (Yang et al. 2012). Jungheim et al. $(2011 b)$ investigated the associations between free fatty acids, COC morphology and ovarian function during IVF and reported that elevated follicular free fatty acid levels are associated with poor COC morphology. These studies corroborate with the present findings and indicate that the lipid composition of follicular fluid has a direct effect on oocyte quality. This study advances our current knowledge by identifying specific fatty acids that are related to oocyte quality. Palmitic acid is the most abundant fatty acid in human follicular fluid (Jungheim et al. 2011a) and was found to be significantly increased in the follicular fluid where the oocytes fertilised but failed to cleave. Excess palmitic acid has been shown to induce apoptosis in human and bovine granulosa cells and impairs their ability for steroidogenesis, which is a critical function for granulosa cells in supporting ovarian follicular development and oocyte maturation (Mu et al. 2001, Vanholder et al. 2006).
Moreover, studies have shown that maturation of oocytes in medium supplemented with elevated levels of the SFA palmitic acid or stearic acid impairs post-fertilisation development (Leroy et al. 2005, Aardema et al. 2011, Van Hoeck et al. 2011). In contrast to the negative effects reported in other studies (Matorras et al. 1998, Kim et al. 2001), stearic acid was found to be significantly increased in the follicular fluid from the cleaved group. Stearic acid can be converted to the monounsaturated oleic acid, with good-quality oocytes reported to contain more of this fatty acid (Haggarty et al. 2006, McKeegan \& Sturmey 2011). Recent results have indicated how important the pattern of fatty acids is and the fact that they act in concert: Van Hoeck et al. (2011) demonstrated that exposing bovine ooctyes to physiologically relevant elevated levels of palmitic acid, oleic acid and stearic acid caused significant dysregulation that was not present when the fatty acids were supplemented singly.

A total of eight PUFAs were identified and quantified by GC/MS analysis, with three significantly different between the follicular fluid from the cleaved and the non-cleaved groups. The significant PUFAs were arachidonic acid, its precursors dihomo- $\gamma$-linolenic acid and DHA, which were found to be increased in the follicular fluid from the cleaved group. In general, the PUFAs support a number of key developmental processes in the embryo such as synthesis of eicosanoids, regulation of endocytosis/exocytosis, ion-channel modulation, DNA polymerase inhibition and gene expression (McKeegan \& Sturmey 2011). Arachidonic acid is the major precursor for prostaglandins and also protects human granulosa cells from palmitic acid- and stearic acidinduced apoptosis (Mu et al. 2001). A number of animal studies investigating the effects of PUFA supplementation in the diet increased oocyte quality (Sorbera et al. 2001, Zeron et al. 2002). However, a study

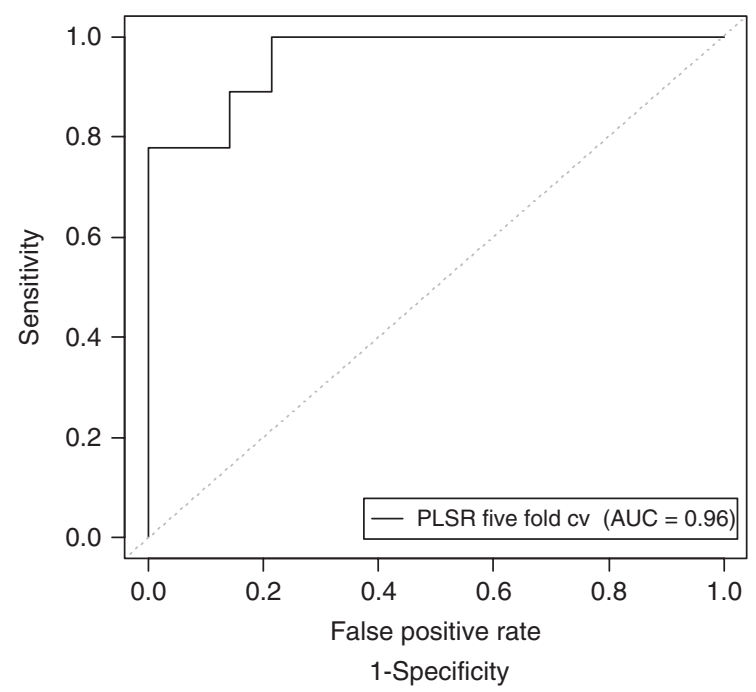

Figure 3 ROC curve produced using the top five metabolites as identified by the random forest (RF) model, with an AUC value of 0.96 . 
investigating the effect of maternal supplementation with a high diet of EPA and DHA found that exposure of murine oocytes to a high n-3 PUFA environment during IVF adversely affected zygote morphology and delayed embryo development (Wakefield et al. 2008). Of interest in our study is the significant increase in the contribution of $n-3$ PUFAs and the alterations in the n-6:n-3 PUFA ratio. A recent review paper on the role of fatty acids in oocyte and early embryo development concluded that different reproductive organs and cells have different proportions of the major saturated, unsaturated and PUFAs and that the maintenance of these ratios is critical for proper development (McKeegan \& Sturmey 2011). Various studies in animal models have demonstrated the potential beneficial effects of these PUFAs (Marei et al. 2010, McKeegan \& Sturmey 2011); however, the experimental evidence in humans to date is lacking. A secondary aim of this study was to apply multivariate statistical analysis to the data in an attempt to identify important fatty acids that could be used as markers to predict oocyte developmental competence (i.e. cleaved/ non-cleaved). The multivariate statistical methods employed resulted in robust models that yielded very good predictive ability. The important metabolites as indicated by the RF model were lignoceric acid, DHA, palmitic acid, stearic acid and arachidic acid. A previous study by Scott et al. (2008) using Raman-based metabolomic profiling of spent culture media developed a viability score that has a diagnostic accuracy of $80.5 \%$ for predicting delivery or failed implantation. Our study resulted with a favourable AUC of 0.96 using the top five important metabolites as indicated by the RF model, highlighting that these fatty acids have a high predictive ability for oocyte developmental competence. An advantage of using GC/MS compared with Raman spectroscopy is the identification of specific metabolites. This study advances our current knowledge by identifying specific fatty acids that are related to oocyte quality.

Our previous work revealed a decrease in levels of lactate and choline/phosphocholine and an increase in levels of glucose and HDL in follicular fluid where the fertilised oocyte failed to cleave (Wallace et al. 2012). In comparison, the models produced in this study indicate that the fatty acid composition of follicular fluid may be more predictive of oocyte developmental competence compared with other biomarkers.

In conclusion, this is the first study to profile extensively the fatty acid composition of human follicular fluid. The fatty acid profiles were significantly different between the follicular fluid from follicles where oocytes fertilised and developed into early cleavage stage embryos and oocytes that fertilised normally but failed to cleave. In addition, RF models identified specific fatty acids that may have the potential to be used as a panel of biomarkers for oocyte quality in the future. Although these results are promising, some limitations in the study exist such as the small sample size. Future studies in larger cohorts are required in order to validate the current findings. Further studies to compare the fatty acid composition of follicular fluid from oocytes where the resultant embryo implanted and resulted in a live birth or failed to implant are also required in order to assess whether the fatty acids identified could predict embryo viability as well as oocyte developmental competence. In addition, due to the increasing evidence of the importance of follicular fluid metabolite composition on oocyte developmental competence, it is necessary to understand the main influencing factors and how its composition may be modulated to improve oocyte quality.

\section{Declaration of interest}

The authors declare that there is no conflict of interest that could be perceived as prejudicing the impartiality of the research reported.

\section{Funding}

This study is supported by grants from the Irish Council for Science and Engineering (IRCSET) and a Science Foundation Ireland (SFI) grant to the Reproductive Biology Research Cluster.

\section{Acknowledgements}

The authors would like to kindly thank all the participants in the study and the staff of the Merrion Fertility Clinic.

\section{References}

Aardema H, Vos PL, Lolicato F, Roelen BA, Knijn HM, Vaandrager AB, Helms BJ \& Gadella BM 2011 Oleic acid prevents detrimental effects of saturated fatty acids on bovine oocyte developmental competence. Biology of Reproduction 85 62-69. (doi:10.1095/biolreprod.110.088815)

Bedair M \& Sumner LW 2008 Current and emerging mass-spectrometry technologies for metabolomics. Trends in Analytical Chemistry 27 238-250. (doi:10.1016/j.trac.2008.01.006)

Berker B, Kaya C, Aytac R \& Satiroglu H 2009 Homocysteine concentrations in follicular fluid are associated with poor oocyte and embryo qualities in polycystic ovary syndrome patients undergoing assisted reproduction. Human Reproduction 24 2293-2302. (doi:10.1093/ humrep/dep069)

Bligh EG \& Dyer WJ 1959 A rapid method of total lipid extraction and purification. Canadian Journal of Biochemistry and Physiology 37 911-917. (doi:10.1139/o59-099)

Botros L, Sakkas D \& Seli E 2008 Metabolomics and its application for noninvasive embryo assessment in IVF. Molecular Human Reproduction 14 679-690. (doi:10.1093/molehr/gan066)

Breiman L 2001 Random forests. Machine Learning 45 5-32. (doi:10.1023/ A:1010933404324)

Edwards RG 1974 Follicular fluid. Reproduction 37 189-219. (doi:10.1530/ jrf.0.0370189)

Fan Y, Murphy TB \& Watson RW 2009 digeR: a graphical user interface R package for analyzing 2D-DIGE data. Bioinformatics 25 3033-3034. (doi:10.1093/bioinformatics/btp514)

Haggarty P, Wood M, Ferguson E, Hoad G, Srikantharajah A, Milne E, Hamilton M \& Bhattacharya S 2006 Fatty acid metabolism in human preimplantation embryos. Human Reproduction 21 766-773. (doi:10.1093/humrep/dei385) 
Jungheim ES, Louden ED, Chi MM, Frolova AI, Riley JK \& Moley KH 2011a Preimplantation exposure of mouse embryos to palmitic acid results in fetal growth restriction followed by catch-up growth in the offspring. Biology of Reproduction 85 678-683. (doi:10.1095/biolreprod.111. 092148)

Jungheim ES, Macones GA, Odem RR, Patterson BW, Lanzendorf SE, Ratts VS \& Moley KH $2011 b$ Associations between free fatty acids, cumulus oocyte complex morphology and ovarian function in vitro fertilization. Fertility and Sterility 95 1970-1974. (doi:10.1016/j.fertnstert.2011.01.154)

Kim JY, Kinoshita M, Ohnishi M \& Fukui Y 2001 Lipid and fatty acid analysis of fresh and frozen-thawed immature and in vitro matured bovine oocytes. Reproduction 122 131-138. (doi:10.1530/rep.0.1220131)

Kovalevsky G \& Patrizio P 2005 High rates of embryo wastage with use of assisted reproductive technology: a look at the trends between 1995 and 2001 in the United States. Fertility and Sterility 84 325-330. (doi:10.1016/j.fertnstert.2005.04.020)

Lei Z, Huhman D \& Sumer LW 2011 Mass spectrometry strategies in metabolomics. Journal of Biological Chemistry 286 25435-25442. (doi:10.1074/jbc.R111.238691)

Leroy JL, Vanholder T, Mateusen B, Christophe A, Opsomer G, de Kruif A, Genicot G \& Van Soom AV 2005 Non-esterified fatty acids in follicular fluid of dairy cows and their effect on developmental capacity of bovine oocytes in vitro. Reproduction 130 485-495. (doi:10.1530/rep.1.00735)

Levay PF, Huyser C, Fourie FL \& Rossouw DJ 1997 The detection of blood contamination in human follicular fluid. Journal of Assisted Reproduction and Genetics 14 212-217. (doi:10.1007/BF02766112)

Marei WF, Wathes DC \& Fouladi-Nashta AA 2010 Impact of linolenic acid on bovine oocyte maturation and embryo development. Reproduction 139 979-988. (doi:10.1530/REP-09-0503)

Matorras R, Ruiz JI, Mendoza R, Ruiz N, Sanjurjo P \& RodriguezEscudero FJ 1998 Fatty acid composition of fertilization-failed human oocytes. Human Reproduction 13 2227-2230. (doi:10.1093/humrep/13. 8.2227)

McKeegan PJ \& Sturmey RG 2011 The role of fatty acids in oocyte and early embryo development. Reproduction, Fertility, and Development $\mathbf{2 4}$ 59-67. (doi:10.1071/RD11907)

de Mouzon J, Goossens V, Bhattacharya S, Castilla JA, Ferraretti AP, Korsak V, Kupka M, Nygren KG \& Nyboe Andersen A 2010 Assisted reproductive technology in Europe, 2006: results generated from European registers by ESHRE. Human Reproduction 25 1851-1862. (doi:10.1093/humrep/deq124)

Mtango NR, Potireddy S \& Latham KE 2008 Oocyte quality and maternal control of development. International Review of Cell and Molecular Biology 268 223-290.

Mu YM, Yanase T, Nishi Y, Tanaka A, Saito M, Jin CH, Mukasa C, Okabe T, Nomura M, Goto K et al. 2001 Saturated FFAs, palmitic acid and stearic acid, induce apoptosis in human granulosa cells. Endocrinology $\mathbf{1 4 2}$ 3590-3597. (doi:10.1210/en.142.8.3590)

Nagy ZP, Sakkas D \& Behr B 2008 Non-invasive assessment of embryo viability by metabolomic profiling of culture media ("metabolomics"). Reproductive Biomedicine Online 17 502-507. (doi:10.1016/S14726483(10)60236-2)

Nagy ZP, Jones-Colon S, Roos P, Botros L, Greco E, Dasig J \& Behr B 2009 Metabolomic assessment of oocyte viability. Reproductive Biomedicine Online 18 219-225. (doi:10.1016/S1472-6483(10)60259-3)

Norli HR, Christiansen A \& Holen B 2010 Independent evaluation of a commercial deconvolution reporting software for gas chromatography mass spectrometry analysis of pesticide residues in fruits and vegetables. Journal of Chromatography. A 1217 2056-2064. (doi:10.1016/j.chroma. 2010.01.022)

Piñero-Sagredo E, Nunes S, de los Santos MJ, Celda B \& Esteve V 2010 NMR metabolic profile of human follicular fluid. NMR in Biomedicine 23 485-495. (doi:10.1002/nbm.1488)

Revelli A, Piane LD, Casano S, Molinari E, Massobrio M \& Rinaudo P 2009 Follicular fluid content and oocyte quality: from single biochemical markers to metabolomics. Reproductive Biology and Endocrinology 7 40. (doi:10.1186/1477-7827-7-40)

Rienzi L, Vajta G \& Ubaldi F 2011 Predictive value of oocyte morphology in human IVF: a systematic review of the literature. Human Reproduction Update 17 34-45. (doi:10.1093/humupd/dmq029)
Robker RL, Akison LK, Bennett BD, Thrupp PN, Chura LR, Russell DL, Lane M \& Norman RJ 2009 Obese women exhibit differences in ovarian metabolites, hormones and gene expression compared to moderateweight women. Journal of Clinical Endocrinology and Metabolism 94 1533-1540. (doi:10.1210/jc.2008-2648)

Scott R, Seli E, Miller K, Sakkas D, Scott K \& Burns DH 2008 Noninvasive metabolomic profiling of human embryo culture media using Raman spectroscopy predicts embryonic reproductive potential: a prospective blinded pilot study. Fertility and Sterility 90 77-83. (doi:10.1016/j. fertnstert.2007.11.058)

Sinclair KD, Lea RG, Rees WD \& Young LE 2007 The developmental origins of health and disease: current theories and epigenetic mechanisms. Reproduction 64 425-443.

Singh R \& Sinclair KD 2007 Metabolomics: approaches to assessing oocyte and embryo quality. Theriogenology 68 56-62. (doi:10.1016/j.theriogenology.2007.04.007)

Sorbera LA, Asturiano JF, Carrillo M \& Zanuy S 2001 Effects of polyunsaturated fatty acids and prostaglandins on oocyte maturation in a marine teleost, the European sea bass (Dicentrarchus labrax). Biology of Reproduction 64 382-389. (doi:10.1095/biolreprod64.1.382)

Swain JE \& Pool TB 2008 ART failure: oocyte contributions to unsuccessful fertilization. Human Reproduction Update 14 431-446. (doi:10.1093/ humupd/dmn025)

Van Hoeck V, Sturmey RG, Bermejo-Alvarez P, Rizos D, Gutierrez-Adan A, Leese HJ, Bols PEJ \& Leroy JLMR 2011 Elevated non-esterified fatty acid concentrations during bovine oocyte maturation compromise early embryo physiology. PLOS ONE 6 e23183. (doi:10.1371/journal.pone. 0023183)

Vanholder T, Leroy JL, Van Soom A, Maes D, Coryn M, Fiers T, de Kruif A \& Opsomer G 2006 Effect of non-esterified fatty acids on bovine theca cell steriodogenesis and proliferation in vitro. Animal Reproduction Science 92 51-63. (doi:10.1016/j.anireprosci.2005.05.014)

Vergouw CG, Botros LL, Roos P, Lens JW, Schats R, Hompes PG, Burns DH \& Lambalk CB 2008 Metabolomic profiling by near-infrared spectroscopy as a tool to assess embryo viability: a novel, non-invasive method for embryo selection. Human Reproduction 23 1499-1504. (doi:10.1093/humrep/den111)

Vergouw CG, Kieslinger DC, Kostelijk HE, Botros LL, Schats R, Hompes PG, Sakkas D \& Lambalk CB 2012 Day 3 embryo selection by metabolomic profiling of culture medium with near-infrared spectroscopy as an adjunct to morphology: a randomized controlled trial. Human Reproduction 27 2304-2311. (doi:10.1093/humrep/ des175)

Wakefield SL, Lane M, Schulz SJ, Hebart ML, Thompson JG \& Mitchell M 2008 Maternal supply of $\omega-3$ polyunsaturated fatty acids alter mechanisms involved in oocyte and early embryo development in the mouse. American Journal of Physiology. Endocrinology and Metabolism 294 E425-E343. (doi:10.1152/ajpendo.00409.2007)

Wallace M, Cottell E, Gibney MJ, McAuliffe FM, Wingfield M \& Brennan L 2012 An investigation into the relationship between the metabolic profile of follicular fluid, oocyte developmental potential, and implantation outcome. Fertility and Sterility 97 1078-1084. (doi:10.1016/j.fertnstert. 2012.01.122)

Wu Y-T, Tang L, Cai J, Lu X-E, Xu J, Zhu X-M, Luo Q \& Huang H-F 2007 High bone morphogenic protein-15 level in follicular fluid is associated with high quality oocyte and subsequent embryonic development. Human Reproduction 22 1526-1531. (doi:10.1093/humrep/dem029)

Yang X, Wu LL, Chura LR, Liang X, Lane M, Norman RJ \& Robker RL 2012 Exposure to lipid-rich follicular fluid is associated with endoplasmic reticulum stress and impaired oocyte maturation in cumulus-oocyte complexes. Fertility and Sterility 97 1438-1443. (doi:10.1016/j.fertnstert.2012.02.034)

Zeron Y, Sklan D \& Arav A 2002 Effect of polyunsaturated fatty acid supplementation on biophysical parameters and chilling sensitivity of ewe oocytes. Molecular Reproduction and Development 61 271-278. (doi:10.1002/mrd.1156)

Received 1 May 2013

First decision 24 June 2013

Accepted 25 July 2013 